Piekarska Ewa, Pawlicki Mateusz, Lopuszyńska Anna, Kozioł Magdalena, Krasa Aleksandra, Piecewicz-Szczęsna Halina. Association Between Male fertility and Vitamin D. Journal of Education, Health and Sport. 2021;11(8):322-327. eISSN 2391-8306. DOI http://dx.doi.org/10.12775/JEHS.2021.11.08.035

https://apcz.umk.pl/czasopisma/index.php/JEHS/article/view/JEHS.2021.11.08.035 https://zenodo.org/record/5273646

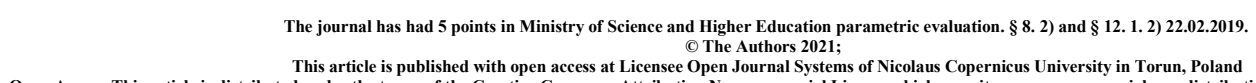

Open Access. This article is distributed under the terms of the Creative Commons Attribution Noncommercial License which permits any noncommercial use, distribution, and reproduction in any medium, provided the original author $(\mathrm{s})$ and source are credited. This is an open access article licensed under the terms of the Creative Commons Attribution Non commercial license Share alike.
(http://creativecommons.org/licenses/by-nc-sa/4.0/) which permits unrestricted, non commercial use, distribution and reproduction in any medium, provided the work is properly cited. The authors declare that there is no conflict of interests regarding the publication of this paper.

Received: 05.08.2021. Revised: 15.08.2021. Accepted: 26.08.2021.

\title{
Association Between Male fertility and Vitamin D
}

\section{Ewa Piekarska ${ }^{1}$, Mateusz Pawlicki ${ }^{1}$, Anna Lopuszyńska ${ }^{1}$, Magdalena Koziol ${ }^{1}$, Aleksandra Krasa ${ }^{1}$, Halina Piecewicz-Szczęsna ${ }^{2}$}

${ }^{1}$ Student Scientific Association at Department of Epidemiology and Clinical Research Methodology Medical University of Lublin, ul. Radziwiłłowska 11, Lublin 20-080, Poland ${ }^{2}$ Department of Epidemiology and Clinical Research Methodology of the Medical University of Lublin, ul. Radziwiłłowska 11, Lublin 20-080, Poland

Corresponding author: Ewa Piekarska, piekarskaewaa@gmail.com

\section{ORCID ID:}

Ewa Piekarska https://orcid.org/0000-0002-4954-379X; piekarskaewaa@gmail.com Mateusz Pawlicki https//orcid.org/0000-0001-8318-6573, pawlak32@gmail.com Anna Łopuszyńska https://orcid.org/0000-0001-5133-4180, lopuszynskaania@gmail.com Magdalena Kozioł https://orcid.org/0000-0002-8671-5968, magdalena.koziol@icloud.com Aleksandra Krasa https://orcid.org/0000-0002-0733-202X,ola.AK62@gmail.com 


\begin{abstract}
Introduction: Infertility affects millions of people of reproductive age worldwide. Male infertility may be caused by a number of different factors including problems in the ejection of semen, absence or low levels of sperm, or abnormal morphology and movement of the sperm. Environmental and lifestyle factors also have a meaningful impact on male reproductive system. Fertility care should include the prevention, diagnosis and treatment of the disorder. That is why numerous studies have explored the role of vitamin D in regulating the functioning of the reproductive system.
\end{abstract}

Aim of study: Investigation of correlation between Vitamin D and male fertility.

Results: Studies consistently show that Vitamin D deficiency has a negative influence on male fertility. Number of trials show that the mean value of Vitamin D serum levels tend to be significantly lower in infertile men compared to fertile men. Vitamin D insufficiency and deficiency has been noticed to be associated with poor semen quality (lower sperm concentrations, lower motility). It became evident that vitamin $\mathrm{D}$ exhibits positive correlation with the male fertility by maintaining the levels of sex hormones, down regulating oxidative stress and up regulating oxidative defence. Supplementation of Vitamin D may improve the quality and function of sperm by increasing its motility and concentration.

Conclusions: Although Vitamin D supplementation cannot be considered for treatment of male infertility itself, physicians should take the vitamin serum levels under consideration while providing treatment for infertile male patients.

Key words: vitamin D; male fertility; infertility; male reproductive system

\title{
Introduction:
}

Infertility is a disease of the male or female reproductive system described as the failure to achieve a pregnancy after 12 months or more of regular unprotected sexual intercourse [1]. Between 48 million couples and 186 million individuals live with infertility globally [2], with male infertility accounting for $40 \%$ of that number [3]. Infertility is influenced by a number of different factors. In the male reproductive system, it is most frequently caused by problems with ejection of semen, absence or low sperm levels, or abnormal morphology and movement of the sperm. Fertility is also affected by environmental factors such as: obesity, smoking, excessive alcohol intake, exposure to pollution and toxins $[4,5]$.

Fertility care should include prevention, diagnosis and treatment of the disorder. That is why numerous studies have explored the role of vitamin D in regulating the functioning of the reproductive system. 
Vitamin D belongs to the family of steroid hormones that has a crucial role in calcium and phosphate homeostasis [6]. It is also known to be effective on glucose metabolism, cellular growth, and immune functions. Deficiency of vitamin D (less than 20 $\mathrm{ng} / \mathrm{mL}$ ) and insufficiency of vitamin $\mathrm{D}(20-29 \mathrm{ng} / \mathrm{mL})$ may be cause of insulin resistance, diabetes, autoimmune disease, cancer, cardiovascular disease, chronic pain, as well as polycystic ovary syndrome and endometriosis in women $[7,8]$.

The presence of vitamin D receptors (VDR) and the enzymes involved in activation and inactivation of vitamin D in germ cells, Leydig cells, epididymis, prostate, seminal vesicles, and mature spermatozoa suggests a direct regulatory role of vitamin $\mathrm{D}$ in the male reproductive system [9]. Concluding, deficiency of the vitamin may be one of the factors influencing male fertility.

\section{Review of available research:}

Studies consistently show that Vitamin D (VD) deficiency has a negative influence on male fertility.

In Vitamin D endemic deficient area study measured and compared 25OHD serum concentrations of 116 fertile and 114 infertile male patients. Infertile group was aditionally divided into 2 subgroups: patients with Vitamin D serum levels above 20ng/mL, and patients with VD serum levels below 20ng/mL. Semen parameters were analysed in infertile patients according to WHO guidelines. Results showed that the mean value of VD serum levels were higher in fertile men. Semen examination showed that infertile patients with VD levels above 20ng/mL had significantly better sperm counts (morphology and movement), compared to those with VD levels $<20 \mathrm{ng} / \mathrm{mL}$ [10].

Study carried out in 112 fertile and 95 infertile Iranian men included semen and venous blood samples analyses. Trial showed that Vitamin D deficiency was significantly higher in infertile men compared to the fertile control group. However there were no significant semen parameters differences between groups [11].

Serum vitamin D is associated with semen parameters and serum testosterone levels according to Inari M Ciccone et al. The study outcomes show mean serum concentrations of VD were significantly lower in men with seminal abnormalities compared to normozoospermic male. VD serum concentrations were also observed to have positive correlation with sperm concentration, total number of spermatozoa and strict morphology [12].

Similar results were given by Elham Azizi et al. study which concluded that Vitamin D has an effect on motility and morphology of spermatozoa, and its lower serum content may affect infertile men [13].

The study carried out in Iraqi fertile and infertile men assessed correlation between vitamin D, testosterone and semen quality. Study included infertile group: 37 patients with teratozoospermia, 34 infertile normozoospermia and 17 control group patients. Seminal fluid and serum samples were collected to address biochemical changes in: Vitamin D levels, VDR, testosterone, parathyroid hormone, proteamine enzyme, zinc, and calcium. Results showed a significant decrease of $\mathrm{VD}$, calcium and zinc in infertile group compared to control. Additional parathormone and protamine levels increased. There was no statistically relevant difference in testosterone and VDR levels between groups [14]. 
Association between Vitamin D, reproductive hormones and sperm parameters in infertile men were also observed in Rehana Rehman et al. study. Fertile and infertile male were divided into subgroups with normal and abnormal sperm parameters. The median values of total sperm count motility, morphology and serum 25OHD were significantly higher in both the fertile and infetile group with normal sperm parameters compared to abnormal sperm parameters group [15].

2018 study by Fahad Hussain et al. assessed homeostatic relevance of Vitamin D in maintaining male fertility. During trial serum samples were collected to compare sex hormones, antioxidant defense and oxidative stress markers in infertile and healthy individuals. As a result it became evident that vitamin $\mathrm{D}$ exhibits positive correlation with the male fertility by maintaining the levels of sex hormones, down regulating oxidative stress and up regulating oxidative defence [16].

Significant association between 25OHD and sperm motility was detected in study among Turkish men. Two sperm samples, with 15 day difference were collected from 33 fertile and 60 infertile men. Undermicrosopic analysis of motility and morphology were carried out according to 2010 WHO criteria. Venous blood samples were collected to measure levels of reproductive system hormones and 25OHD levels. In infertile patients semen volume and VD levels were significantly lower. Study shows positive correlation between Vitamin D levels and total progressive motile sperm count [17].

Study by Kadiliya Jueraitetibaike et al. evaluated association of semen quality with both serum and seminal plasma vitamin D levels and its underlying mechanisms. The study detected positive correlation between seminal plasma and sperm kinetic parameters, stating that VD may be involved in sperm motility by promoting activation of the sperm mitochondrial respiratory chain to produce ATP (adesinotriphosporan) and increase intracellular calcium concentrations [18].

As the Vitamin D role in male reproductive health became more relevant many of available publications investigated the influence of Vitamin D supplementation on male fertility.

A. Alzoubi et al. study searched for association between VD and idiopathic infertility and whether supplementation can restore semen quality parameters. Trial enrolled 117 men: 30 healthy, fertile men plus 20 secondary infertile men as control groups, and a test group of 67 idiopathic infertile men (divided into low VD serum level, and normal VD serum level groups). Idiopathic infertile men with Vitamin D deficiency were supplemented with oral VD $5000 \mathrm{IU}$ for a month. After month-long supplementation there was a relevant improvement of progressive and total sperm motility in the treated group [19].

Effects of VD on apoptosis and sperm quality in asthenozoospermia were adresses in Mahin Taheri Moghadam et al. trial. Study included 80 semen samples of asthenozoospermic and healthy men to analyze sperm motility, morphology, apoptosis and necrosis and chromatin integrity. Samples were divided into control and experimental groups (which recieved $20 \mu \mathrm{Mol}$ of VD). The addition of VD has resulted in rise of motile sperm number, rise of progresive motility and reduced apoptosis and necrosis. However there were no morphology changes noticed [20]. 
According to Martin Blomberg Jensen et al. study Vitamin D supplementation was not associated with changes in semen parameters. However spontaneous pregnancies tended to be higher in couples in which man was in the treatment group during trial (which received initially $300,000 \mathrm{IU}$, then $1400 \mathrm{IU}$ cholecalciferol and $500 \mathrm{mg}$ of calcium daily for 150 days) compared to the control group which received placebo [21].

Impact of supplementation was observed in study in insufficient/ deficient infertile men with oligozoospermia and asthenozoospermia. Patients received 60,000 IU cholecalciferol and $500 \mathrm{mg}$ daily calcium supplementation for a period of 6 months. Outcomes showed improvement in sperm concentrations and progresive sperm motility in infertile men with oligozoospermia [22].

\section{Conclusions:}

Low levels of serum Vitamin D could be a risk factor of poor semen quality in infertile men. Review of available studies presents relevant information that vitamin D serum levels have a direct and positive correlation with semen quality and male reproductive potential. Due to inadequacy of appropriate randomization and controls in mentioned trials Vitamin D supplementation cannot be considered for treatment of male infertility itself. However it may improve the quality and function of sperm by increasing its motility and concentration. Physicians should take the vitamin serum levels under consideration while providing treatment for infertile male patients. Further research on the subject is needed as infertility is a complex disorder.

\section{References:}

1. World Health Organization (WHO). International Classification of Diseases, 11th Revision (ICD-11) Geneva: WHO 2018

2. Rutstein SO, Shah IH. Infecundity infertility and childlessness in developing countries. Geneva: World Health Organization 2004

3. Kumar N, Singh AK. Trends of male factor infertility, an important cause of infertility: A review of literature. J Hum Reprod Sci. 2015;8(4):191-196. doi:10.4103/0974-1208.170370

4. Gore AC, Chappell VA, Fenton SE, et al. EDC-2: The Endocrine Society's Second Scientific Statement on Endocrine-Disrupting Chemicals. Endocrine Reviews 2015;36(6):E1-E150. doi: 10.1210/er.2015-1010

5. Segal TR, Giudice LC. Before the beginning: environmental exposures and reproductive and obstetrical outcomes. Fertility and Sterility 2019;112(4):613-21.

6. Boisen IM, Bøllehuus Hansen L, Mortensen LJ, Lanske B, Juul A, Blomberg Jensen M. Possible influence of vitamin D on male reproduction. J Steroid Biochem Mol Biol. 2017;173:215-222. doi:10.1016/j.jsbmb.2016.09.023

7. Blomberg Jensen M, Gerner Lawaetz J, Andersson AM, et al. Vitamin D deficiency and low ionized calcium are linked with semen quality and sex steroid levels in infertile men. Hum Reprod. 2016;31(8):1875-1885. doi:10.1093/humrep/dew152

8. Gorkem U, Ozturk Inal Z, Inal H. Assessment of the Relationship Between Serum Vitamin D Levels and Obesity in the Reproductive-Aged Women. EJMO 2019;3(1):43-48.

9. Lorenzen M, Boisen IM, Mortensen LJ, Lanske B, Juul A, Blomberg Jensen M. Reproductive endocrinology of vitamin D. Mol Cell Endocrinol. 2017;453:103-112. doi:10.1016/j.mce.2017.03.023 
10. Akhavizadegan H, Karbakhsh M. Comparison of Serum Vitamin D between Fertile and Infertile Men in A Vitamin D Deficient Endemic Area: A Case-Control Study. Urologia Journal. 2017;84(4):218-220. doi:10.5301/uj.5000248

11. Shahraki Z, Mojahed BS, Shahraki A. Comparison of Vitamin D Levels in Fertile and Infertile Men. Maedica (Bucur). 2020;15(1):96-98. doi:10.26574/maedica.2020.15.1.96

12. Ciccone IM, Costa EM, Pariz JR, et al. Serum vitamin D content is associated with semen parameters and serum testosterone levels in men. Asian $J$ Androl. 2021;23(1):52-58. doi:10.4103/aja.aja_9_20

13. Azizi E, Naji M, Shabani-Nashtaei M, Aligholi A, Najafi A, Amidi F. Association of serum content of 25-hydroxy vitamin $\mathrm{D}$ with semen quality in normozoospermic and oligoasthenoteratozoospermic men. Int J Reprod Biomed. 2018;16(11):689-696.

14. Albaldawy, Mustafa \& Alsallami, Alaauldeen. (2017). Study of association among vitamin D, testosterone and semen quality in fertile and Iraqi infertile men. Journal of Pharmaceutical Sciences and Research. 9. 1067-1071.

15. Rehman R, Lalani S, Baig M, Nizami I, Rana Z, Gazzaz ZJ. Association Between Vitamin D, Reproductive Hormones and Sperm Parameters in Infertile Male Subjects. Front Endocrinol (Lausanne). 2018;9:607. Published 2018 Oct 16. doi:10.3389/fendo.2018.00607

16. Fahad Hussain, Arif Malik, Muhammad Saeed, Muhammad Imran, Sulayman Waquar, Hassan Shafique, et al. Homeostatic relevance of vitamin D in maintaining male fertility in human: Down-regulation of oxidative stress and up-regulation of anti-oxidative defense and steroidal hormones. Asian Pac J Reprod 2018; 7(2): 56-61.

17. Inal, HA, Ozturk Inal, Z, Mermer, S, Aksoy, E, Bayraktar, AM, Can, U. Investigation of serum vitamin D and ischaemia-modified albumin levels in infertile Turkish men. Andrologia. 2020; 52:e13507.

18. Jueraitetibaike K, Ding Z, Wang DD, et al. The effect of vitamin D on sperm motility and the underlying mechanism. Asian J Androl. 2019;21(4):400-407. doi:10.4103/aja.aja_105_18

19. Alzoubi A, Mahdi H, Al Bashir S, et al. Normalization of serum vitamin d improves semen motility parameters in patients with idiopathic male infertility. Acta Endocrinol (Buchar). 2017;13(2):180-187. doi:10.4183/aeb.2017.180

20. Moghadam MT, Hosseini G, Absalan F, Tabar MH, Nikbakht R. Effects of Vitamin D on Apoptosis and Quality of Sperm in Asthenozoospermia [published online ahead of print, $2020 \mathrm{Jul} 14$ ]. JBRA Assist Reprod. 2020;24(3):316-323. doi:10.5935/1518-0557.20200009

21. Blomberg Jensen M, Lawaetz JG, Petersen JH, Juul A, Jørgensen N. Effects of Vitamin D Supplementation on Semen Quality, Reproductive Hormones, and Live Birth Rate: A Randomized Clinical Trial. J Clin Endocrinol Metab. 2018 Mar 1;103(3):870-881. doi: 10.1210/jc.2017-01656. PMID: 29126319.

22. Wadhwa L, Priyadarshini S, Fauzdar A, Wadhwa SN, Arora S. Impact of Vitamin D Supplementation on Semen Quality in Vitamin D-Deficient Infertile Males with Oligoasthenozoospermia. J Obstet Gynaecol India. 2020;70(1):44-49. doi:10.1007/s13224-01901251-1 